$551.501 .6: 519.27$

\title{
Statistical Method of Estimating Isolines
}

\author{
by \\ M. Ogawara and H. Yamazaki \\ Meteorological Research Institute \\ (Received July 28, 1952)
}

\begin{abstract}
In this paper, it is pointed out firstly that the so-called objective method of drawing isolines which is based on the method of least squares can be generalized but that it is disadvantageous in practice. As an alternative, a method based on the simultaneous confidence region of true value at each station is proposed. What has the objective meaning is the confidence region by which the existence region of isolines is provided. Especially, the confidence interval of proportional division point of two adjacent stations can be easily found by a graphical method. A definite set of isolines may be drawn arbitrarily in the restricted limit, by considering several points in each local area successively. The method is developed for two cases; the one concerns the distribution of mean values and the other the directly observed values. In the former the only assumption is that every set of random fluctuations of simultaneously observed values submits to the same multivariate normal probability distribution law, and in the latter the assumption that the covariances of observed values are known must be added.
\end{abstract}

\section{Introduction}

Scientific conclusions on the syroptic meteorology must be based on an objective analysis, and in the synoptic weather analysis isolines of various quantities play an important role. As an objective method of drawing isolines, H. A. PANofsky [1] adopted the method of least squares. In this paper, after criticizing PANoFsiky's method (Section 2), we shall give an alternative method of estimating isolines.

Let $a=f(\xi, \eta)$ be a function of two independent variables $\xi, \eta$. Then a set of isolines which is an intuitional representation of the function is given by

$$
f(\xi, \eta)=c_{j} \quad(j=0, \pm 1, \pm 2, \ldots \ldots),
$$

where $c_{j}(j=0, \pm 1, \ldots \ldots)$ are constants and usually taken as $c_{j}=c_{0}+j \Delta c$. In research 
works, such as the above mentioned drawing of weather chart, we must estimate the function or its isolines by the observations at a finite number of points $\left(\xi_{i}, \eta_{i}\right)$ $(i=1,2, \ldots \ldots, k)$

$$
x_{i}=a_{i}+z_{i} \quad(i=1,2, \ldots \ldots, k),
$$

where $a_{i}=f\left(\xi_{i}, \eta_{i}\right)$ and $z_{i}$ is the error of observation which is due to measuring instruments, method of observation and other complicated causes.

In the above case we are concerned with directly observed values. However, in mean weather chart and climatic chart, for instance, we treat the distribution of mean values. In this case we may assume that a function $a=f(\xi, \eta ; t)$ is a stationary stochastic process with time parameter $t$ and the mean value function to be estimated is that of the process, i. e.

$$
\bar{a}=\bar{f}(\xi, \eta)=E f(\xi, \eta ; t) .
$$

The estimation of a set of isolines

$$
\bar{f}(\xi, \eta)=c_{j} . \quad(j=0, \pm 1, \pm 2, \ldots \ldots)
$$

should be done, in this case, by the observations

$$
\begin{gathered}
x_{i t}=f\left(\xi_{i}, \eta_{i} ; t\right)+z_{i t}=\bar{f}\left(\xi_{i}, \eta_{i}\right)+y_{i t}+z_{i t}, \quad(t=1,2, \ldots \ldots, n) \\
\bar{x}_{i}=\bar{a}_{i}+z_{i}^{*} \quad(i=1,2, \ldots \ldots, k),
\end{gathered}
$$

where $\bar{a}_{i}=\bar{f}\left(\xi_{i}, \eta_{i}\right), \bar{x}_{i}=\sum_{i=1}^{n} x_{i t} / n, z_{i}^{*}=\sum_{t=1}^{n} y_{i t} / n+\sum_{t=1}^{n} z_{i t} / n$ and where $y_{t t}$ is the random fluctuation of the process (the real phenomena) itself and $z_{i t}$ is the error of observation.

In general, $z_{i}(i=1,2, \ldots \ldots, k)$ in (2) as well as $z_{i}^{*}(i=1,2, \ldots \ldots, k)$ in (5) are correlated to each other. In Sections 3 and 4 of this paper we shall treat the case (5) and in Section 5 the case (2).

\section{On the so-called objective method of drawing isolines}

As an objective method, H. A. PANofsky [1] adopted a method of least squares by which a function $a=f(\xi, \eta)$ is directly estimated. In his method, firstly,

I. functional form of $f\left(\xi, \eta ; \theta_{1}, \theta_{2}, \ldots \ldots\right)$ must be assumed, where $\theta_{i} \quad(i=1$, $2, \ldots .$.$) are unknown parameters which are to be estimated by the observed data.$ For instance, let us suppose that we wish to estimate the height of constant pressure surface $f\left(\xi, \eta ; \theta_{1}, \theta_{2}, \ldots ..\right)$. For the radiosonde stations $\left(\xi_{i}, \eta_{i}\right)(i=1$, $\left.2, \ldots \ldots, k_{0}\right)$ and the pilot-balloon stations $\left(\xi_{j}{ }^{\prime}, \eta_{j}^{\prime}\right)\left(j=k_{0}+1, \ldots \ldots, k\right)$ we put

and

$$
\begin{aligned}
f_{i} & =f\left(\xi_{i}, \eta_{i} ; \theta_{1}, \theta_{2}, \ldots \ldots\right) & & i=1,2, \ldots \ldots, k_{0} \\
& =0 & & i=k_{0}+1, \ldots \ldots, k \\
f^{\prime}{ }_{j} & =f\left(\xi_{j^{\prime}}^{\prime} \eta_{j}^{\prime} ; \theta_{1}, \theta_{2}, \ldots . .\right) & & j=k_{0}+1, \ldots \ldots, k
\end{aligned}
$$

respectively. Let $x_{i}$ be the observed height at $\left(\xi_{i}, \eta_{i}\right)\left(i=1,2, \ldots \ldots, k_{0}\right)$ and let

$$
x_{j}=v_{j} \cdot \nabla f^{\prime} ; \quad\left(j=k_{0}+1, \ldots \ldots, k\right),
$$

where $v_{j}$ is the wind velocity vector observed at $\left(\xi^{\prime}{ }_{j}, \eta^{\prime}{ }_{j}\right)$. Then the unknown parameters $\theta_{1}, \theta_{2}, \ldots .$. may be estimated, by the method of least squares, so as to minimize the sum. 


$$
S=\sum_{i=1}^{k} w_{i}\left(x_{i}-f_{i}\right)^{2}
$$

where $w_{i}$ is the weight of observed value $x_{i}$. Thus, in this method, the following second assumption is necessary :

II. The weight $w_{i}$ 's $\left(\sum_{i=1}^{k} w_{i}=1\right)$ or the ratios of variances $\sigma_{i}=\sigma^{2} / w_{i}(i=1,2, \ldots \ldots$, $k$ ) are known, where $\sigma^{2}$ is a proportional constant.

In the above formulation as well as in PANOFsry's method it is assumed that the errors of $x_{i}(i=1,2, \ldots \ldots, k)$ are independent from each other, but if

II'. the ratios of covariances $r_{i j} \sigma_{i} \sigma_{j}$ of $x_{i}$ and $x_{j}(i, j=1, \ldots \ldots, k)$ are known, the method of least squares is likewise applicable; that is; the sum which should be minimized is written as

$$
S=\sum_{i, j=1}^{k} w_{i j}\left(x_{i}-f_{i}\right)\left(x_{j}-f_{j}\right)
$$

where $\left(w_{i j}\right)=\sigma^{2}\left(r_{i j} \sigma_{i} \sigma_{j}\right)^{-1}\left(\sigma^{2}\right.$ is a proportional constant $)$.

Further, even if the position $\left(\xi_{i}, \eta_{i}\right)$ of observation has a random error, the method of least squares is also applicable, provided that

$\mathrm{I}_{1}$. the type of functional relation $a=f\left(\alpha, \beta ; \theta_{1}, \theta_{2}, \ldots \ldots\right)$ is known, where $\alpha$ and $\beta$ are the expected values of $\xi$ and $\eta$ respectively, that is, $(\alpha, \beta)$ is the true pasition corresponding to $(\xi, \eta)$,

and that

$\mathrm{II}_{1}$. for three dimensional variables $\left(x_{i}, \xi_{i}, \eta_{i}\right)$ an assumption similar to that of the above II or II' holds.

If the probability distribution of errors is Gaussian (or normal), the estimation by the above method has a probabilistic meaning. When the functional form is not linear with respect to unknown parameters, the estimation procedures may be carried out by Deming's method [2]. For the estimation of mean value function $\bar{f}(\xi, \eta)$ a similar formulation holds.

Thus the method of least squares offers a tool of fairly general utility for our purpose. However, a serious disadvantage of this method lies, we think, in the assumption I. For instance, let us consider the case when we take a polynomial

$$
a=f(\xi, \eta)=\sum_{i, j} \theta_{i j} \xi^{i} \eta^{j} \text {. }
$$

In order that this function should satisfactorily represent the real state, the order of this polynomial must be so large that $x_{i}-a_{i}(i=1,2, \ldots \ldots, k)$, where $a_{i}=f\left(\xi_{i}, \eta_{i}\right)$, are all considered to be random error, that is, the $k$-dimensional point $\left(a_{1}, \ldots \ldots, a_{k}\right)$ must lie in the confidence ellipsoid with suitably selected confidence coefficient. In general, in order to estimate faithfully the real state of distribution, it may be recessary to assume a functional form $f(\xi, \eta)$ with a fairly large number of unknown parameters the estimation procedure of which requires a great deal of labour. and may be almost prohibitable for routine work.

The so-called objective analysis based. on the method of least squares may be a so: $t$ of point estimation of isolines. We shall propose an alternative method in the 
following sections, which may be said to be a method of interval estimation of isolines.

\section{Isolines for mean values}

If we assign a functional form $f(\xi, \eta)$, the most probable (maximum likelihood) estimate of a set of isolines is uniquely determined. However, in general, there may be an innumerable set of isolines each of which is admissible as a representation of true distribution so long as the information is limited in the observed data. We wish to know the existence region of such admissible isolines.

Firstly, let us consider the distribution of mean value. Let the observed values at the $i$ th station be $x_{i t}(t=1,2, \ldots \ldots, n), i=1,2, \ldots \ldots, k$, then the sample mean and the sample variance are

$$
\bar{x}_{i}=\sum_{t=1}^{n} x_{i t} / n \text { and } s_{i}{ }^{2}=\sum_{i=1}^{n}\left(x_{i t}-\bar{x}_{i}\right)^{2} / n \quad(i=1,2, \ldots \ldots, k)
$$

respectively. Now, we may assume about the probability distribution of the random factor $y_{i t}+z_{i t}$ in (4) as follows.

Assumption: For every $t, x_{i t}(i=1,2, \ldots \ldots, k)$ are independently distributed according to the same $k$-dimensio al normal distribution

$$
(2 \pi)^{-k / 2} A^{1 / 2} \exp \left[-\frac{1}{2} \sum_{i, j=1}^{k} A_{i j}\left(x_{i t}-a_{i}\right)\left(x_{j t}-a_{j}\right)\right],
$$

where $a_{i}=f\left(\xi_{i}, \eta_{i}\right)$ is the expected value (true value) of $x_{i t},\left(A_{i j}\right)$ is the inverse matrix of the covariance matrix of $x_{i t}(i=1,2, \ldots \ldots, k)$ and $A=\operatorname{det}\left(A_{i j}\right)$. (In what follows we need not assume anything about the true value of $A_{i j}$.)

Then the confidence region for $\left(a_{1}, a_{2}, \ldots \ldots, a_{k}\right)$ with confidence coefficient $1-\alpha$ is given by the interior of hyper-ellipsoid

$$
\frac{(n-k) n}{k} \sum_{i, j=1}^{k} s^{i j}\left(\bar{x}_{i}-a_{i}\right)\left(\bar{x}_{j}-a_{j}\right)=F_{n-k}^{k}(\alpha) \quad(k<n),
$$

where

$$
\begin{aligned}
& \left(s^{i j}\right)=\left(n r_{i j} s_{i} s_{j}\right)^{-1}, \\
& r_{i j}=\frac{1}{n} \sum_{t=1}^{n}\left(x_{i t}-\bar{x}_{i}\right)\left(x_{j t}-\bar{x}_{j}\right) / s_{i} s_{j}
\end{aligned}
$$

and $F_{n-k}^{k}(\alpha)$ is the $100 \alpha \%$ point of $F$-distribution with $k$ and $n-k$ degrees of freedom $[3]^{*}$. The equation (7) shows that to what extent the differences $\bar{x}_{i}-a_{i}$ can be allowed to get, where $a_{i}$ is the value at the $i$ th station which is obtained by interpolation under a supposed set of isolines. But the formula (7) may not be penetrating and the restriction $k<n$ seems to limit its applicability. In connection with these points, we put

$$
u=\left|\bar{x}_{i}-a_{i}\right| \sqrt{n} / s_{i} \quad(i=1,2, \ldots \ldots, k)
$$

and observe the following special cases.

* The formula (7) is easily derived from the distribution of Hotelling's generalized Student. ratio. M. Masuyama used this formula in 1943. 
i) The case where $r_{i j} \equiv r(i, j=1,2, \ldots ., k)^{*}$. In this case (3. 2) becomes as follows,

$$
\begin{aligned}
\sum_{i} \frac{\left(\bar{x}_{i}-a_{i}\right)^{2}}{s_{i}{ }^{2}}-\frac{2 r}{1+(k-2) r} & \sum_{i<j} \frac{\left(\bar{x}_{i}-a_{i}\right)\left(\bar{x}_{j}-a_{j}\right)}{s_{i} s_{j}} \\
& =\frac{k(1-r)[1+(k-1) r]^{\prime}}{(n-k)[1+(k-2) r]} F_{n-k}^{k}(\alpha)
\end{aligned}
$$

Substituting (9) into this equation, we have

$$
u^{2}=\frac{n k(1-r)[1+(k-1) r]}{(n-k)\left[k+\left(k^{2}-2 k+2 h\right) r\right]} F_{n-k}^{k}(\alpha)
$$

where

$$
\begin{aligned}
h= & \left(\text { the number of negative }\left(\bar{x}_{i}-a_{i}\right)\left(\bar{x}_{j}-a_{j}\right)\right) \\
& \left.- \text { the number of positive }\left(\bar{x}_{i}-a_{i}\right)\left(\bar{x}_{j}-a_{i}\right)\right) .
\end{aligned}
$$

If $k \rightarrow \infty$ and if then $k / n \rightarrow p<1$ and $h / k \rightarrow 0$,

$$
u^{2} \rightarrow \frac{1-r}{1-p}
$$

ii) The case where $r_{i j}=r^{|i-j|}(0<r<1)$. In this case

$$
\begin{aligned}
\frac{\left(\bar{x}_{1}-a_{1}\right)^{2}}{s_{1}{ }^{2}} & +\left(1+r^{2}\right) \sum_{i=2}^{k-1} \frac{\left(\bar{x}_{i}-a_{i}\right)^{2}}{s_{i}^{2}}+\frac{\left(\bar{x}_{k}-a_{k}\right)^{2}}{s_{k}{ }^{2}} \\
& -2 r_{i=1}^{k-1} \frac{\left(\bar{x}_{i}-a_{i}\right)\left(\vec{x}_{i+1}-a_{i+1}\right)}{s_{i} s_{i+1}}=\frac{\left(1-r^{2}\right) k}{n-k} F_{n-k}^{k}(\alpha)
\end{aligned}
$$

and we have

$$
u^{2}=\frac{n k(1-r)}{(n-k)\left[k+(k-2) r^{2}+2 h r\right]} F_{n-k}^{k}(\alpha) .
$$

If $k \rightarrow \infty$ and if then $k / n \rightarrow p<1$ and $h / k \rightarrow q$,

$$
u^{2} \longrightarrow \frac{1-r^{2}}{\left(1+r^{2}+2 q r\right)(1-p)} \text {. }
$$

Table 1 shows some values of $u$ for (11) and (14). The means of $h$ for the cases of $k=2$ and $k=3$ are illustrated in Fig. 1 .

In Table 1 we observe that the value of $u$ does not remarkably decrease as $k$ increases barring the case where $h$ is negative and the correlation $r$ is large. $u$ rather increases when $k / n$ approaches to one, which fact is also seen for large $n$ from (12) and (15). Therefore, we see that, in general, even if we take many points (stations) simultaneously into consideration the precision of the estimation of isolines does not necessarily increase; thus it may be sufficient to consider two or three points in each local area successively. For comparison, if we individually estimate the true value $a_{i}$ at each point, its confidence limits with confidence coefficient $1-\alpha$ is given by

* This is not a restriction on the sample variables (observed values), but we suppose the case where we got a special sample satisfying this condition. 
Table 1. Values of $u=\left|\overrightarrow{x_{i}}-\ddot{a}_{i}\right| \sqrt{n} / s_{i} \quad(i=1,2, \ldots \ldots, k) ; 1-\alpha=95 \%$.

(1) The case where $r_{i j} \equiv r$.

\begin{tabular}{|c|c|c|c|c|c|c|c|c|c|c|c|c|c|}
\hline \multirow{2}{*}{\multicolumn{2}{|c|}{$n$}} & \multicolumn{4}{|c|}{$r=0$} & \multicolumn{4}{|c|}{$r=0.5$} & \multicolumn{4}{|c|}{$r=0.9$} \\
\hline & & 2 & 3 & 9 & $n-5$ & 2 & 3 & 9 & $n-5$ & 2 & 3 & 9 & $n-5$ \\
\hline$\rightarrow$ & 10 & 2.36 & 2.49 & 49.09 & 3.18 & 1.67 & 1.84 & 36.08 & 2.37 & 0.75 & 0.84 & 16.28 & 1.08 \\
\hline 11 & 30 & 1.89 & 1.81 & 1.84 & 5.21 & 1.34 & 1.34 & 1.35 & 3.75 & 0.61 & 0.61 & 0.61 & 1.68 \\
\hline-5 & 50 & 1.82 & 1.71 & 1.61 & 6.67 & 1.29 & 1.28 & 1.18 & 4.77 & 0.57 & 0.57 & 0.53 & 2.13 \\
\hline $\begin{array}{c}7 \\
11 \\
s\end{array}$ & $\begin{array}{l}10 \\
30 \\
50\end{array}$ & $\begin{array}{l}2.36 \\
1.89 \\
1.82\end{array}$ & & & & $\begin{array}{l}2.89 \\
2.32 \\
2.23\end{array}$ & & & & $\begin{array}{l}3.26 \\
2.65 \\
2.51\end{array}$ & & & \\
\hline$\stackrel{m}{11}$ & $\begin{array}{l}10 \\
30 \\
50\end{array}$ & & & $\begin{array}{r}49.09 \\
1.84 \\
1.61\end{array}$ & $\begin{array}{l}3.18 \\
5.21 \\
6.67\end{array}$ & & & $\begin{array}{r}35.40 \\
1.33 \\
1.16\end{array}$ & $\begin{array}{l}2.21 \\
3.74 \\
4.76\end{array}$ & & & $\begin{array}{r}15.52 \\
0.58 \\
0.51\end{array}$ & $\begin{array}{l}0.98 \\
1.68 \\
2.13\end{array}$ \\
\hline$m$ & 10 & & 2.49 & 49.09 & 3.18 & & 3.53 & 38.00 & 2.82 & & 4.25 & 17.01 & 1.33 \\
\hline 1 & 30 & & 1.81 & 1.84 & 5.21 & & 2.56 & 1.43 & 3.78 & & 3.09 & 0.64 & 1.69 \\
\hline$\stackrel{\|}{11}$ & 50 & & 1.73 & 1.61 & 6.67 & & 2.42 & 1.24 & 4.78 & & 2.91 & 0.56 & 2.14 \\
\hline
\end{tabular}

(2) The case where $r_{i j}=\boldsymbol{r}^{|i-j|}$

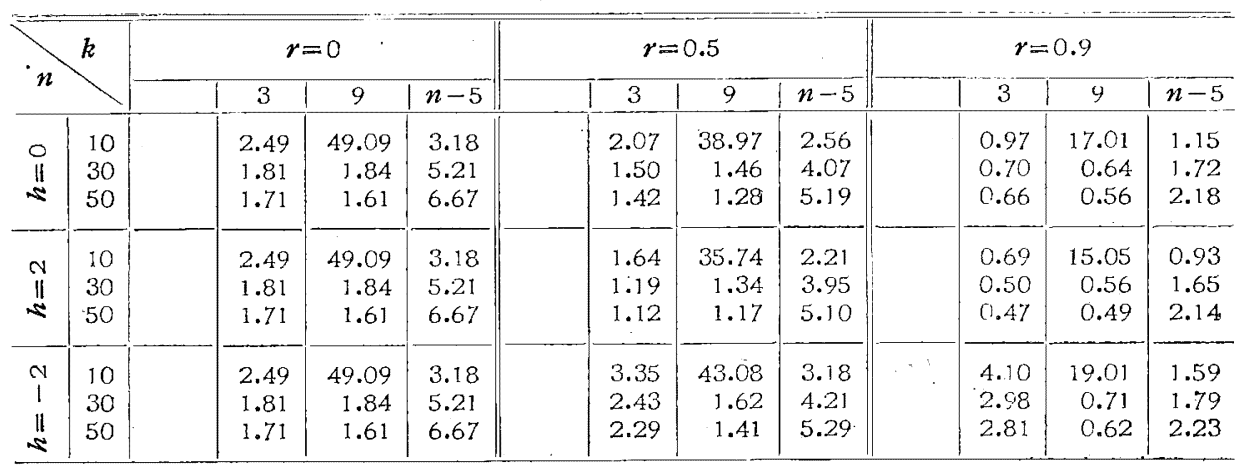
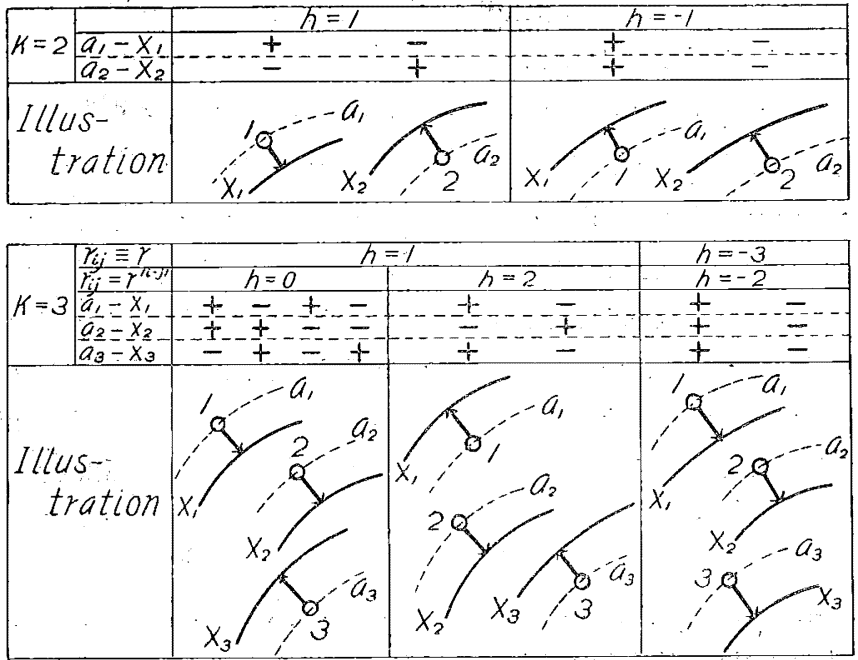

Fig. 1.

The relation between observed values and the corresponding isolines restricted by $h$. Full line $\left(x_{i}\right)$ is the isoline with the observed value at the ith point. Doțted line $\left(a_{i}\right)$ is the isoline through the ith point. 


$$
\bar{x}_{i} \pm \sqrt{\frac{n}{n-1}} t_{n-1}(\alpha) s_{i}
$$

where $t_{n-1}(\alpha)$ is the positive $\alpha / 2$ point of the $t$-distribution with $n-1$ degrees of freedom. The values of the coefficient $\sqrt{n /(n-1)} \cdot t_{n-1}(\alpha)$ are given in Table 2.

An interval estimation by confidence region does not assign a specially fixed set of isolines, but in our research work we need a set of definitely drawn isolines. A practical procedure of drawing a probable isoline may be formulated as follows:

i) Plot $\bar{x}_{i} \pm s_{i} / \sqrt{n}$ for each point. If possible, it will be desirable to write the correlation coefficient $r_{i j}$ between adjacent points on the Table 2. Values of $\sqrt{\frac{n}{n-1}} \cdot t_{n-1}(\alpha)$ line connecting them.

ii) Draw a trial set of isolines by considering two or three adjacent points and by referring to the interval $\bar{x}_{i} \pm u s_{i} / \sqrt{n}$, for successive local regions, where the value of $u$ can be selected in the column $k=2$ or $k=3$ in Table 1 (if we take $1-\alpha=95 \%$ ) according to the type of isolines illustrated in Fig. 1.

iii) Read the value $a_{i}$ for the $i$ th point by the above drawn trial set of isolines and test if $\left|\bar{x}_{i}-a_{i}\right| \leqq u s_{i} / \sqrt{n}$ or not. If not, some correction will be necessary.

Table 3 is an example of the test of propriety of an assumed set of isolines in the western part of Chiba prefecture in Fig. 3.

Table 3. An example of the test of propriety of an assumed set of isolines for the monthly mean temperature for January in the Western part of Chiba prefecture $(1916 \sim 25)$.

$n=10, k=3,1-\alpha=95 \%$ and we assumed that $r=0.9$

\begin{tabular}{|c|c|c|c|c|c|c|}
\hline \multirow{2}{*}{ Station } & \multirow{2}{*}{$\begin{array}{c}\bar{x}_{i} \\
\text { Observed } \\
\text { mean value }\end{array}$} & \multirow{2}{*}{$\frac{s_{i}}{\sqrt{n}}$} & \multirow{2}{*}{$\begin{array}{l}a_{i} \\
\text { Read by } \\
\text { isolines }\end{array}$} & \multirow{2}{*}{$\bar{x}_{i}-a_{i}$} & \multicolumn{2}{|c|}{$u s_{i} / \sqrt{n}$} \\
\hline & & & & & $\begin{array}{c}\text { The case of } \\
r_{i j} \equiv r\end{array}$ & $\begin{array}{c}\text { The case of } \\
r_{i j}=r^{|i-j|}\end{array}$ \\
\hline $\begin{array}{l}\text { Anegasaki } \\
\text { Yawata } \\
\text { Chiba }\end{array}$ & $\begin{array}{l}3.0^{\circ} \mathrm{C} \\
3.3 \\
3.8\end{array}$ & $\begin{array}{l}0.41 \\
0.76 \\
0.61\end{array}$ & $\begin{array}{l}3.3^{\circ} \mathrm{C} \\
3.4 \\
3.7\end{array}$ & $\begin{array}{r}-0.3 \\
-0.1 \\
0.1\end{array}$ & $\left.\begin{array}{l}0.34 \\
0.64 \\
0.51\end{array}\right)\left(\begin{array}{c}h=1 \\
u=0.84\end{array}\right)$ & $\left.\begin{array}{l}0.40 \\
0.74 \\
0.59\end{array}\right)\left(\begin{array}{c}h=0 \\
u=0.97\end{array}\right)$ \\
\hline $\begin{array}{l}\text { Yawata } \\
\text { Chiba } \\
\text { Miyako }\end{array}$ & $\begin{array}{l}3.3 \\
3.8 \\
3.0\end{array}$ & $\begin{array}{l}0.76 \\
0.61 \\
0.62\end{array}$ & $\begin{array}{l}3.4 \\
3.7 \\
3.5\end{array}$ & $\begin{array}{r}-0.1 \\
0.1 \\
-0.5\end{array}$ & $\begin{array}{l}0.641 \\
0.51 \\
0.52\end{array}\left(\begin{array}{c}h=1 \\
u=0.84\end{array}\right)$ & $\left.\begin{array}{l}0.52 \\
0.42 \\
0.43\end{array}\right\}\left(\begin{array}{c}h=2 \\
u=0.69\end{array}\right)$ \\
\hline
\end{tabular}

If we wish to adopt a more rigorous procedure, we must use the equation (7) itself. For $k=3$, it is reduced to

$$
\sum_{i . j=1}^{3} a_{i j} t_{i} t_{j}=R^{2}
$$


where $t_{i}=\left(\bar{x}_{i}-a_{i}\right) \sqrt{ } \bar{n} / s_{i}$ and

$$
\begin{aligned}
& a_{11}=\left(1-r_{23}^{2}\right) / \Delta, \quad a_{22}=\left(1-r^{2}{ }_{13}\right) / \Delta, \quad a_{33}=\left(1-r_{12}^{2}\right) / \Delta, \\
& a_{12}=\left(r_{31} r_{32}-r_{12}\right) / \Delta, \quad a_{23}=\left(r_{12} r_{13}-r_{23}\right) / \Delta, \quad a_{31}=\left(r_{23} r_{21}-r_{31}\right) / \Delta, \\
& \Delta=1+2 r_{12} r_{23} r_{31}-r_{12}^{2}-r_{23}^{2}-r^{2}{ }_{31}, \\
& R^{2}=\frac{3 n}{n-3} F_{n-3}{ }_{n-3}(\alpha)
\end{aligned}
$$

and if we put $u=\left|t_{i}\right|(i=1,2,3)$

$$
u=R\left[\sum_{i, j=1}^{3} a_{i, j}\right]^{-1 / 2}
$$

The foregoing method is comparatively simple but somewhat technical. If it is admitted to draw an isoline by considering only two adjacent points, we can derive a more definite method which will be stated in the following section.

\section{Confidence interval of proportional division point}

Let the observed value at two stations $P_{1}$ and $P_{2}$ be $x_{1}$ and $x_{2}$ respectively. As a simple method of drawing an isoline $f(\xi, \eta)=c$, a linear inverse interpolation (or extrapolation) has been generally used by which the line is so drawn that it passes though the point $P_{12}$ dividing the segment $\bar{P}_{1} P_{2}$ in proportion to the ratio $\left(c-x_{1}\right)$ : $\left(x_{2}-c\right)$. If the observed values $x_{1}$ and $x_{2}$ have any random error the division point is also a random variable.

Again let us consider the case of isolines for mean values and derive the confidence interval of the division point under the assumption $(6)(k=2)$. Let $P_{1}$ be

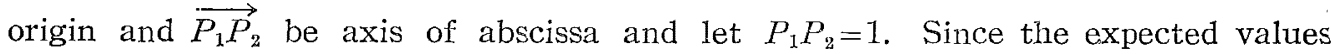
(true value) of $\bar{x}_{1}$ and $\bar{x}_{2}$ are $a_{1}$ and $a_{2}$ respectively, the abscissa $\xi$ of true division point is obtained from the equation of straight line

$$
y=a_{1}+\left(a_{2}-a_{1}\right) \xi ;
$$

by putting $y=c$;

$$
\xi=\left(c-a_{1}\right) /\left(a_{2}-a_{1}\right) .
$$

Now, according to $(7)$, the confidence region of $\left(a_{1}, a_{2}\right)$ with confidence coefficient $1-\alpha$ is given by the ellipse

$$
A\left(a_{1}-\bar{x}_{1}\right)^{2}+2 H\left(a_{1}-\bar{x}_{1}\right)\left(a_{2}-\bar{x}_{2}\right)+B\left(a_{2}-\bar{x}_{2}\right)^{2}=R^{2},
$$

where

$$
\left.\begin{array}{l}
A=1 / s_{1}{ }^{2}, \quad B=1 / s_{2}{ }^{2}, \quad H=-r / s_{1} s_{v}, \\
R^{2}=\frac{2\left(1-r^{2}\right)}{n-2} F_{n-2}^{2}(\alpha),
\end{array}\right\}
$$

provided that $r$ is the sample correlation coefficient between $x_{1 t}$ and $x_{2 t}(t=1,2, \ldots \ldots$, $n)$. When the point $\left(a_{1}, a_{3}\right)$ moves along the circumference of ellipse (21), the envelope of straight line (19) is obtained as a hyperbola: 


$$
\begin{aligned}
y & =\bar{x}_{1}+\left(\bar{x}_{2}-\bar{x}_{1}\right) \xi \pm R \sqrt{\frac{A \xi^{2}-2 H \xi(1-\xi)+B(1-\xi)^{2}}{A B-H^{2}}} \\
& =\bar{x}_{1}+\left(\bar{x}_{2}-\bar{x}_{1}\right) \xi \pm \sqrt{\frac{2}{n-F^{2}{ }^{2}{ }_{n-2}(\alpha)} \sqrt{{s_{2}}^{2} \xi^{2}+2 r s_{1} s_{2}} \xi(1-\xi)+{s_{1}}^{2}(1-\xi)^{2}} .
\end{aligned}
$$

Using this we can get the confidence interval of $\xi$ as follows.

First Method. Draw the hyperbola (23). Let the intersection of straight line $y=c$ with the hyperbola be $\left(\xi_{1}, \xi_{2}\right)\left(\xi_{1}\right.$ may be $-\infty$ and $\xi_{2}$ may be $\left.+\infty\right)$. Then the $\left(\xi_{1}, \xi_{2}\right)$ is the required confidence interval.

The abscissas $\xi_{1}$ and $\xi_{2}$ can be also obtained as follows. Let the point $\left(a_{1}, a_{2}\right)$ move along the circumference of ellipse $(21)$, then $\xi$ gets its extreme value when

$$
\left\{\begin{array}{c}
\left.a_{1}=\bar{x}_{1} \pm R \sqrt{Q^{2} /\left(A Q^{2}-2 H P Q+B P^{2}\right.}\right), \\
a_{2}=\bar{x}_{2} \mp R \sqrt{P^{2} /\left(A Q^{2}-2 H P Q+B P^{2}\right)},
\end{array}\right.
$$

where

$$
\left\{\begin{array}{l}
P=A\left(c-\bar{x}_{1}\right)+H\left(c-\bar{x}_{2}\right) \\
Q=H\left(c-\bar{x}_{1}\right)+B\left(c-\bar{x}_{2}\right)
\end{array}\right.
$$

Accordingly,

$$
\left(a_{2}-\bar{x}_{2}\right) /\left(a_{1}-\bar{x}_{1}\right)=-\sqrt{P^{2}} / \sqrt{Q^{2}} .
$$

While, from (21) we have

Hence,

$$
\frac{d a_{2}}{d a_{1}}=-\frac{A\left(a_{1}-\bar{x}_{1}\right)+H\left(a_{2}-\bar{x}_{2}\right)}{H\left(a_{1}-\bar{x}_{1}\right)+B\left(a_{2}-\bar{x}_{2}\right)} .
$$

$$
\frac{a_{2}-\bar{x}_{2}}{a_{1}-\bar{x}_{1}}=\left(\frac{d a_{2}}{d a_{1}}\right)_{a_{1}=a_{2}=c .}
$$

Thus we may go through the following procedure:

Second Method: (i) Draw the ellipse (21). (ii) Draw a tangent to the ellipse at the intersection point of the ellipse with the straight line connecting two points $(c$, c) and $\left(\bar{x}_{1}, \bar{x}_{2}\right)$. (iii) Let the coordinates of both endpoints of diameter of the ellipse parallel to the tangent be $\left(a_{1}, a_{2}\right)$ and $\left(a_{1}{ }^{\prime}, a_{2}{ }^{\prime}\right)$. (iv) Let the abscissa of intersection of the straight line $y=c$ with another straight line connecting two points $\left(0, a_{1}\right)$ and $\left(1, a_{2}\right)$ be $\xi_{1}$ and let the abscissa of intersection of $y=c$ with the straight line connecting $\left(0, a_{1}{ }^{\prime}\right)$ and $\left(1, a_{2}{ }^{\prime}\right)$ be $\xi_{2}$. Then $\xi_{1}$ and $\xi_{2}$ are the required confidence limits of division point $\xi$ with confidence coefficient $1-\alpha$.

The confidence interval thus obtained will be easily transferred on the straight line in question; for instance, in a mean weather chart, by proportional relation. Two examples are given in Fig. 2. In drawing an isoline $f(\xi, \eta)=c$, we may select, in general, pairs of stations $\left(P_{i}, P_{j}\right)$ such that they are as near as possible to each other and $\bar{x}_{i}<c<\bar{x}_{j}$ or $\bar{x}_{i}>c>\bar{x}_{j}$. It is easily seen that, if the two stations $P_{i}$ and $P_{j}$ were far apart from each other, the confidence interval of the division point would become very broad and the assumption that the function $f(\xi, \eta)$ is linear in such a wide area could not be admitted. 
(a) Sanrizuka-Yökaichiba
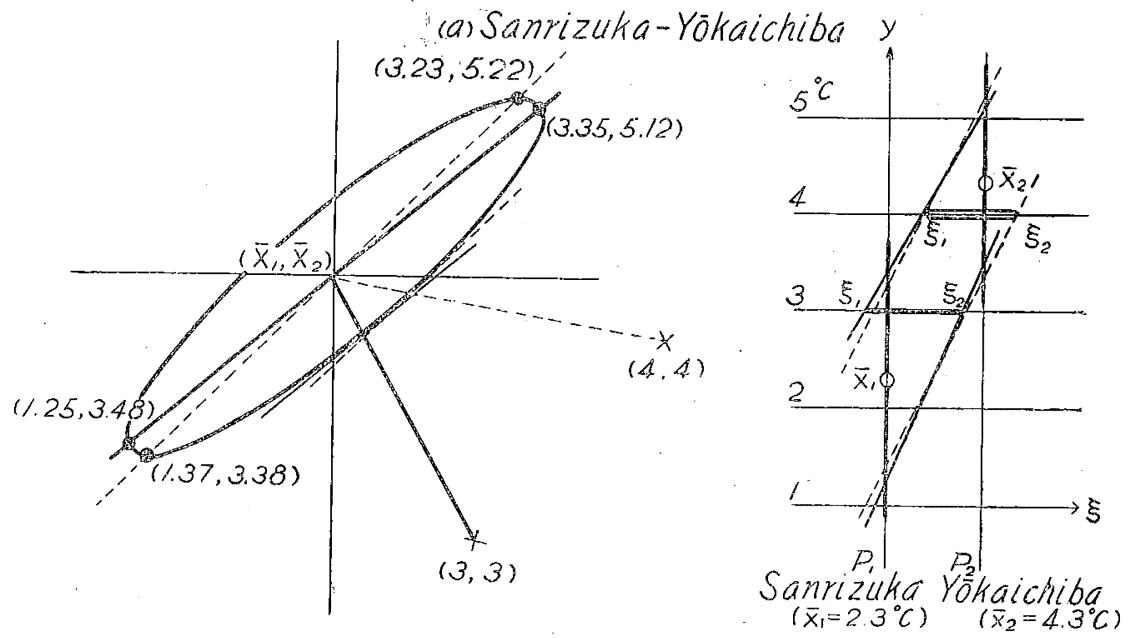

(b) Huma-Asahi

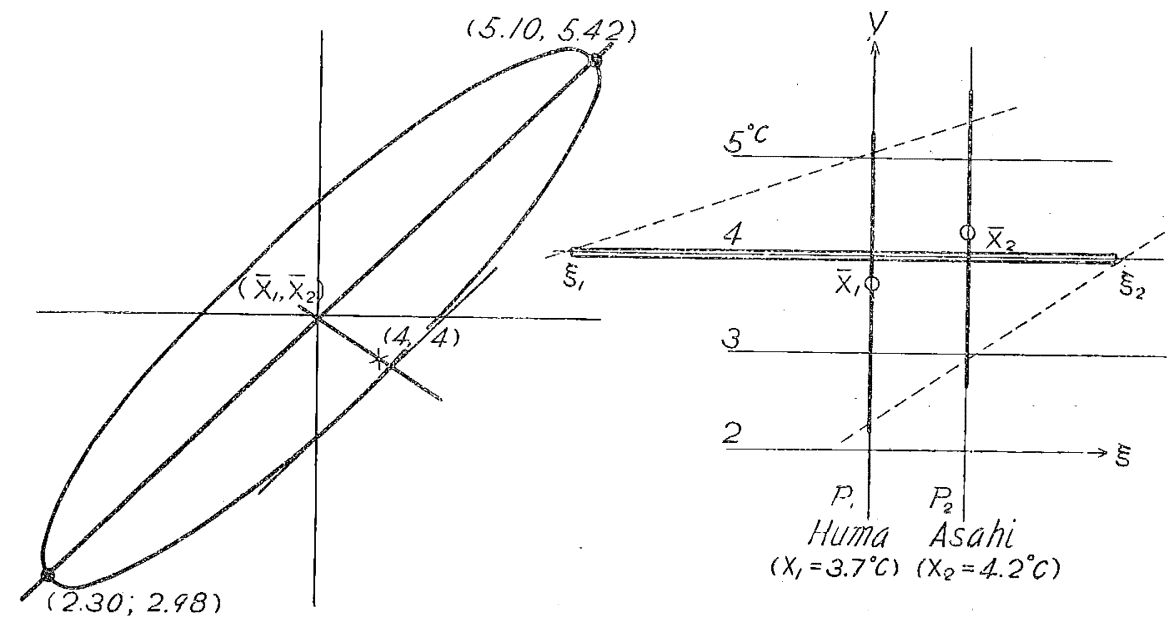

Fig. 2. Confidence interval of proportional division point. Confidence coefficient is $80 \%$.

Fig. 3 is an illustration in which the above method was applied to some part (cf. Fig. 2) and to the other part (except several stations) the method stated in the previous section was applied (cf. Table 3). Owing to the shortage of observed years, the reliability of isolines in Fig. 3 is very low. The correlation coefficients of representative pairs of stations in Fig. 3 are shown in Table 4.

When the period of observation varies with stations and the observations are not always simultaneous, to the estimation of isolines for mean values, as a rule, the method for the case of direct observation, which will be stated in the next section, must be applied. In fact, this is done for several stations in Fig. 3, since those stations have some lack of observations. 


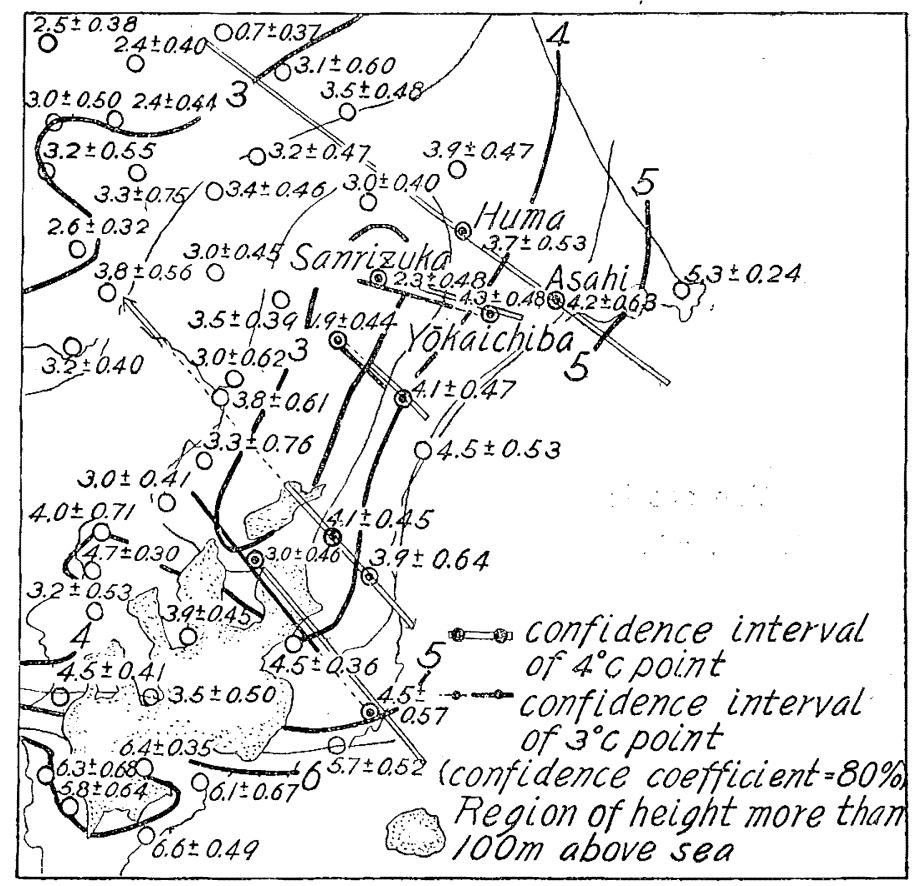

Fig. 3. Mean temperature distribution for January in Chiba prefecture (1901 1930). Observed period is for most stations 1916 1925. It varies in 1901 1930 with several stations in that period the fluctuation of monthly mean temperature can be considered to have no trend in the district.

Table 4. Spatial correlation of monthly mean temperature for January (1916 25).

\begin{tabular}{|c|c|c|}
\hline Stations $\left(\begin{array}{c}\text { Number of } \\
\text { observed years }\end{array}\right)$ & $\begin{array}{l}\text { Correlation } \\
\text { coefficient }\end{array}$ & $\begin{array}{l}\text { Confidence limits } \\
\text { with conf. coef. } 90 \%\end{array}$ \\
\hline Tōkyō-Matsudo & 0.39 & $-0.27, \quad 0.78$ \\
\hline -Fusa & 0.86 & $0.44, \quad 0.96$ \\
\hline -Urayasu & 0.84 & $0.50, \quad 0.95$ \\
\hline -Kisarazu & 0.85 & $0.55, \quad 0.94$ \\
\hline -Ohara & 0.98 & $0.90, \quad 0.99$ \\
\hline Asahi-Fuma & 0.76 & $-0.10, \quad 0.95$ \\
\hline Yökaichiba-Sanrizuka (9) & 0.73 & $0.25, \quad 0.91$ \\
\hline Tógane-Yachimata (10) & 0.97 & $0.89, \quad 0.99$ \\
\hline Mobara-Ichinomiya (9) & 0.84 & $0.48, \quad 0.95$ \\
\hline Ohara-Tsurumai & 0.98 & $0.90, \quad 0.99$ \\
\hline
\end{tabular}


When the periods of observation at two stations $P_{1}$ and $P_{2}$ are different, let the observed values be

$$
\begin{aligned}
& x_{11}, x_{12}, \ldots \ldots, x_{1 m}, x_{1 m+1}^{\prime}, \ldots \ldots x_{1 n_{2}}^{\prime} \quad\left(\text { at } P_{1}\right), \\
& \left.x_{21}, x_{22}, \ldots \ldots, x_{2 m}, x^{\prime}{ }_{2 m+1}, \ldots \ldots x_{2 n_{1}}^{\prime} \quad \text { (at } P_{2}\right),
\end{aligned}
$$

where $x_{1 t}$ and $x_{2 t}$ are simultaneous observations $(t=1,2, \ldots \ldots, m)$ but there is no temporal correspondence between any $x^{\prime}{ }_{1 u}$ and $x^{\prime}{ }_{2 v}\left(u=m+1, \ldots \ldots, n_{1} ; v=m+1, \ldots \ldots\right.$, $n_{2}$ ). Then the correlation coefficient $r^{\prime}$ between average values $\bar{x}_{1}$ and $\bar{x}_{2}$ may be estimated by

$$
r^{\prime}=\frac{m}{\sqrt{n_{1} n_{2}}} r
$$

where $r$ is the correlation coefficient of the pair $\left(x_{1}, x_{2}\right)$ and is estimated by their sample $\left(x_{1 t}, x_{2 t}\right)(t=1, \ldots \ldots, m)$, provided that $x_{1 t}(t=1,2, \ldots \ldots)$ as well as $x_{2 t}(t=1$, $2, \ldots \ldots)$ are independent from each other.

\section{Isolines for directly observed values}

Let $x_{i}(i=1,2, \ldots \ldots, k)$ be the observed value of $a_{i}=f\left(\xi_{i}, \eta_{i}\right)(i=1,2, \ldots \ldots, k)$ respectively. In regard to the random errors $z_{i}=x_{i}-a_{i}(i=1,2, \ldots \ldots, k)$ we assume, firstly, the same assumption as (6);

Assumption I: $\left(x_{1}, \ldots \ldots, x_{k}\right)$ is distributed according to the $k$-dimensional normal probability distribution law

$$
(2 \pi)^{-k / 2} \mathrm{~A}^{1 / 2} \exp \left[-\frac{1}{2} \sum_{i, j=1}^{k} \mathrm{~A}_{i j}\left(x_{i}-a_{i}\right)\left(x_{j}-a_{j}\right)\right],
$$

where $\left(A_{i j}\right)=\left(\rho_{i j} \sigma_{i} \sigma_{j}\right)^{-1}$ and $A=\operatorname{det}\left(A_{i j}\right)$, provided $\sigma^{2}{ }_{i}$ is the variance of $x_{i}$ and $\rho_{i j}$ is the correlation coefficient betweien $x_{i}$ and $x_{j}$. If we add another

Assumption II : $\sigma_{i}$ and $\rho_{i j}(i, j=1,2, \ldots \ldots, k)$ are known, then the confidence region of $\left(a_{1}, \ldots \ldots, a_{k}\right)$ with confidence coefficient $1-\alpha$ is given by the interior of a hyperellipsoid

$$
\sum_{i, j=1}^{k} A_{i j}\left(a_{i}-x_{i}\right)\left(a_{j}-x_{j}\right)=\chi_{i}^{2}(\alpha),
$$

where $\chi_{i}^{2}(\alpha)$ is the $100 \alpha \%$ point of $\chi^{2}$-distribution with $k$ degrees of freedom. The equation (30) corresponds to (7) by a translation

$$
s_{i} / \sqrt{n} \rightarrow \sigma_{i}, \quad r_{i j \rightarrow p_{i},} \quad \frac{n k}{n-k} F^{k}{ }_{n-k_{i}}(\alpha) \rightarrow \chi_{k}^{2}(\alpha) .
$$

Therefore, we see that the arguments in sections 3 and 4 hold for the present case also. If we put, in the equation (30),

$$
v=\left|a_{i}-x_{i}\right| / \sigma_{i}(i=1,2, \ldots \ldots, k),
$$

we have, as an example, the following Table 5 .

We may draw an isoline as we consider that, for several adjacent points, each true value lies, with $100(1-\alpha) \%$ certainty, in the interval 
Table 5. Values of $v=\left|a_{i}-x_{i}\right| / \sigma_{i} \quad(1 \cdots a=95 \%)$

\begin{tabular}{|c|c|c|c|c|c|c|c|c|c|c|c|}
\hline \multicolumn{4}{|c|}{$k=2, \quad \rho_{1 Y}=\rho$} & \multicolumn{4}{|c|}{$k=3, \quad \rho_{i j} \equiv \rho$} & \multicolumn{4}{|c|}{$k=3, \quad \rho i j=\rho^{|i-j|}$} \\
\hline$h \rho$ & 0 & 0.5 & 0.9 & $h \rho$ & 0 & 0.5 & 0.9 & & 0 & 0.5 & 0.9 \\
\hline 1 & 1.73 & 1.22 & 0.55 & 1 & 1.61 & 1.19 & 0.54 & 0 & 1.61 & 1.34 & 0.62 \\
\hline-1 & 1.73 & 2.12 & 2.39 & -3 & 1.61 & 2.28 & 2.70 & $\begin{array}{r}2 \\
-2\end{array}$ & $\begin{array}{l}1.61 \\
1.61\end{array}$ & $\begin{array}{l}1.06 \\
2.17\end{array}$ & $\begin{array}{l}0.45 \\
2.66\end{array}$ \\
\hline
\end{tabular}

(33)

$$
x_{i} \pm v \sigma_{i}
$$

where the value of $v$ may be chosen from such a table as Table 5 . The method of test for propriety of an assumed set of isolines and other details are similar to those in the foregoing sections.

When $k=2$, the equation (30) is reduced to

$$
A\left(a_{1}-x_{1}\right)^{2}+2 H\left(a_{1}-x_{1}\right)\left(a_{2}-x_{2}\right)+B\left(a_{2}-x_{2}\right)^{2}=R^{2},
$$

where

$$
A=1 / \sigma_{1}^{2}, \quad B=1 / \sigma_{2}^{2}, \quad H=-\rho / \sigma_{1} \sigma_{2}, \quad R^{2}=\left(1-\rho^{2}\right) \chi_{2}^{2}(\alpha),
$$

provided $\rho \equiv \rho_{12}$. The envelope of the straight line connecting two points $\left(0, a_{1}\right)$ and $\left(1, a_{2}\right)$

$$
y=a_{1}+\left(a_{2}-a_{1}\right) \xi
$$

is a hyperbola

$$
\begin{aligned}
y & =x_{1}+\left(x_{2}-x_{1}\right) \xi \pm R \sqrt{\frac{A \xi^{2}-2 H \xi(1-\xi)+B(1-\xi)^{2}}{A B-H^{2}}} \\
& =x_{1}+\left(x_{2}-x_{1}\right) \xi \pm \sqrt{\chi_{2}^{2}}(\alpha)\left\{\sigma_{1}^{2}(1-\xi)^{2}+2 \rho \sigma_{1} \sigma_{2} \xi(1-\xi)+\sigma_{2}{ }^{2} \xi^{2}\right\}
\end{aligned}
$$

In the case where we take only two adjacent points inta consideration, we can geometrically draw the confidence interval of their proportional division point by the ellipse (34) or hyperbola (37).

As an example, let us estimate iso-geopotentialmeter-lines of $500 \mathrm{mb}$ pressure surface at 1200 (L.M.T.) Feb. 3, 1950, in the neighbourhood of Japan. We might assume that

$$
\sigma_{1}=\sigma_{2}=\sigma=20 \mathrm{gpm} \text { and } \rho=0 .
$$

Then the confidence ellipse of $\left(a_{1}, a_{2}\right)$ is reduced to a circle

$$
\left(a_{1}-x_{1}\right)^{2}+\left(a_{2}-x_{2}\right)^{2}=\sigma^{2} \chi_{2}^{2}(\alpha) \text {. }
$$

Two examples of graphical solution for confidence interval of proportional division point are shown in Fig. 4. (cf. The Second Methad in section 4 and Fig. 7. Of course the First Method can be applied also.) A trial set of isolines is drawn with the confidence intervals thus obtained in view. (Fig. 7)

Next, let us test by the observations of wind (at $5 \mathrm{~km}$ height), the propriety of the iso-gpm-lines thus drawn. In regard to wind direction we might assume that 

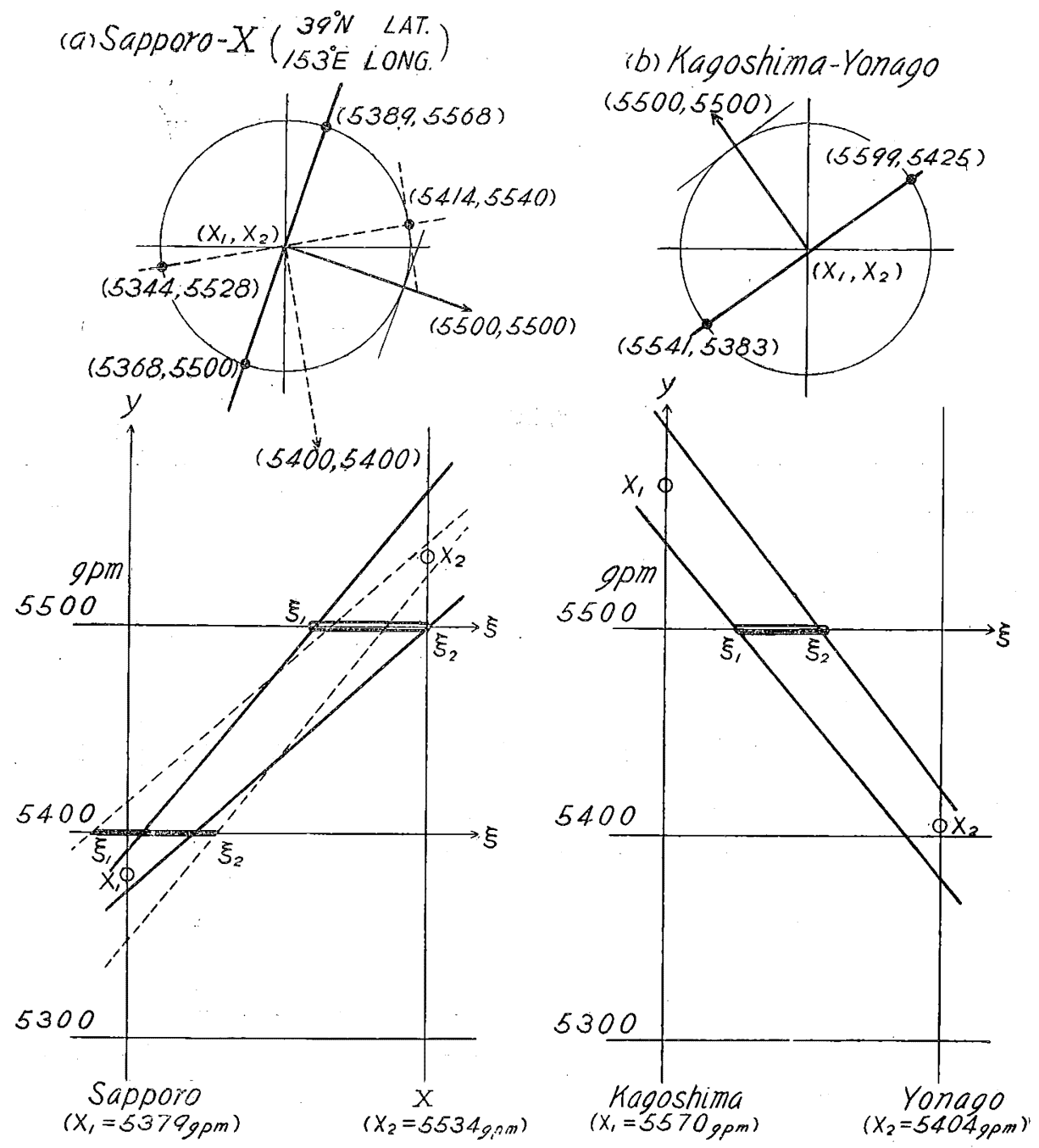

Fig. 4. Confidence interval of consiant gepotential-meter point on the $500 \mathrm{mb}$ constant pressure surface. Conîdence coefficient is $80 \%$.

$$
\sigma_{1}=\sigma_{2}=\sigma=10 \text { (degree) and } \rho=0 .
$$

Then the hyperbola for this case is reduced to

$$
y=x_{1}+\left(x_{2}-x_{1}\right) \xi \pm R^{\prime} \sqrt{\xi^{2}+(1-\xi)^{2}},
$$

where $R^{\prime}=\sqrt{\chi_{2}{ }^{2}(\alpha)} \cdot \sigma$. Let the ordinates of the hyperbola at the intersection of an iso-gpm-line with the segment connecting the two adjacent points where the observations of wind are performed be $\theta_{1}$ and $\theta_{2}$. (Fig. 5) If the direction of an isogpm-line lies inside of the interval $\left(\theta_{1}, \theta_{2}\right)$, we may regard that the iso-gpm-line is adapted for the wind direction locally. (Fig. 7) 
(a) Sendai-Tateno

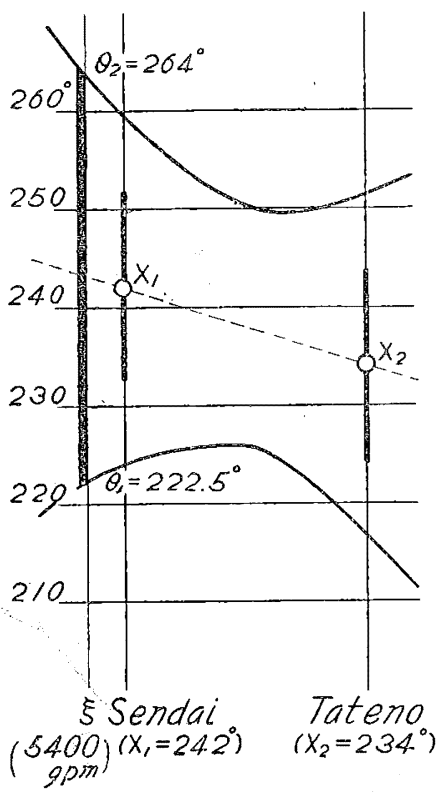

(b) Kagoshima-Tateno

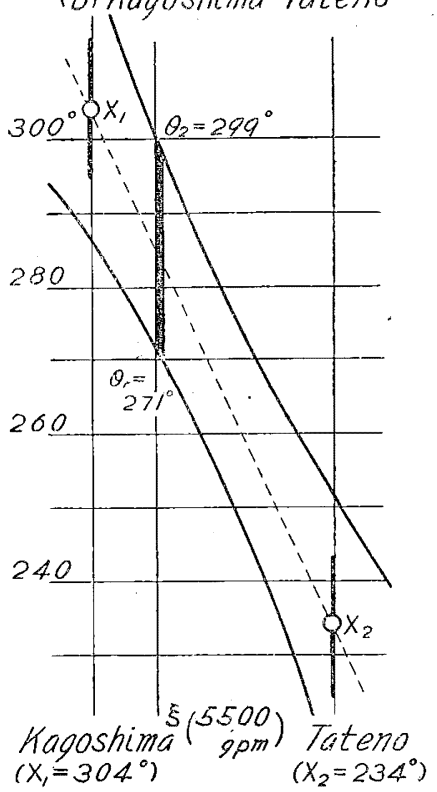

Fig. 5. Conidence region of wind direction on an isogpm-line. Confidence coefficient is $80 \% . \xi$ is a point on the line through two stations intersected by an iso-gpm-line.

Hyperbola: $\quad y=x_{1}+\left(x_{2}-x_{1}\right) \xi \pm R^{\prime} \sqrt{\xi^{2}+(1-\xi)^{2}}$, $R^{\prime}=\sqrt{\chi_{2}{ }^{2}(\alpha)} \cdot \sigma, \quad \alpha=20 \%, \quad \sigma=10^{\circ}$.

As to the wind velocity, in addition to the assumption $\rho=0$, we assume that $\sigma_{i}$ is equal to the integral part of $\left(x_{i} / 10\right) \mathrm{m} / \mathrm{sec}$, where $x_{i}$ $(m / \mathrm{sec})$ is the observed wind velocity at $\left(\xi_{i}\right.$, $\left.\eta_{i}\right)$. Then the equation of hyperbola concerning two points $\left(\xi_{1}, \eta_{1}\right)$ and $\left(\xi_{2}, \eta_{2}\right)$ is

$$
\begin{aligned}
y & =x_{1}+\left(x_{2}-x_{1}\right) \xi \\
& \pm \sqrt{\chi_{2}{ }^{2}(\alpha)} \cdot \sqrt{\sigma_{1}{ }^{2}(1-\xi)^{2}+\sigma_{2}{ }^{2} \xi^{2}} .
\end{aligned}
$$

By the same method as the above, we can find the confidence limits $\left(v_{1}, v_{2}\right)$ of true wind velocity $v$ at the middle point of the segment which is the portion of the straight line connecting two stations that lies between two adjacent iso-gpm-lines in question. (Fig. 6) Now, the confidence limits $\left(d_{1}, d_{2}\right)$ of the dis. tance $d$ of two adjacent iso-gpm-lines are derived from that of wind velocity by the following equation :

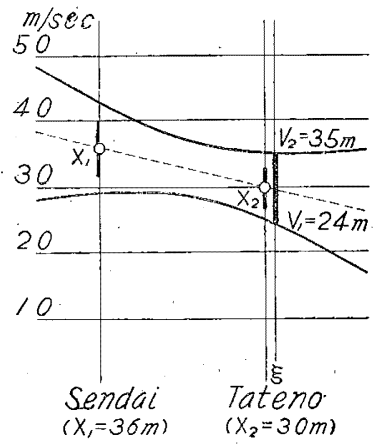

Fig. 6. Confidence interval of wind velocity at an intermediate point between two adjacent iso-gpm-lines. Confidence coefficient is $80 \%$. $\xi$ is the middle point of the segment of a line through Sendai and Tateno between two iso-gpm-lines.

Hyperbola:

$$
\begin{gathered}
y=x_{1}+\left(x_{2}-x_{1}\right) \xi \\
\pm \sqrt{\chi_{2}^{2}(\alpha)\left\{\sigma_{1}^{2}(1-\xi)^{2}+\sigma_{2}^{2} \xi^{2}\right\}} \\
\alpha=20 \%, \quad \sigma_{1}=4 \mathrm{~m} / \mathrm{sec}, \quad \sigma_{2}=3 \mathrm{~m} / \mathrm{sec} .
\end{gathered}
$$




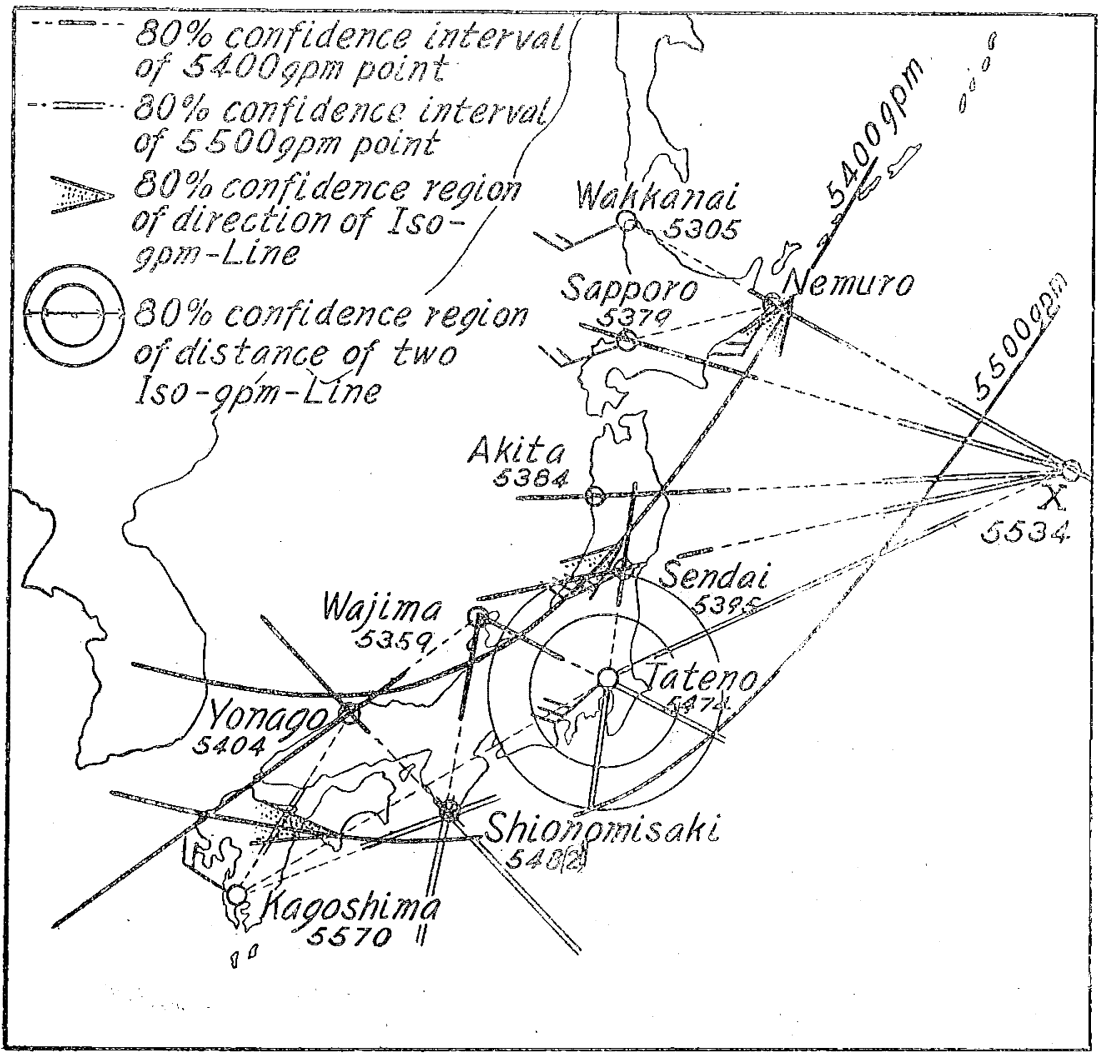

Fig. 7. Iso-gpm-lines on the $500 \mathrm{mb}$ constant pressure surface over the neighbourhood of Japan. Feb. 3, 1200 (L.M.T.), 1950.

$$
d_{j}=\Delta \phi /\left(2 \omega \sin \phi \cdot v_{j}\right) \text { (meter) }(j=1,2),
$$

where, in our case, $\Delta \phi=100 \mathrm{gpm}=1000\left[\mathrm{~m}^{2} / \mathrm{sec}^{-2}\right]$. Diameters of concentric circles in Fig. 7 are $d_{1}$ and $d_{2}$. As, in this example, the two iso-gpm-lines pass through the ring-like region between the concentric circles, the distance of the iso-gpm-lines may be regarded to be pertinent, in the local area, to the wind velocity.

In the above method we treated individually the height of constant pressure surface, wind direction and wind velocity. But, in a more rigorous method, they should be simultaneously considered. However, such a method would be extremely com. plicated since we must then deal with a hyper-ellipsoid as their confidence region.

\section{Additional remarks}

By means of finite number of observations with random errors, we cannot find definitely the true spatial distribution of a quantity or its isolines. In our method of estimation of isolines, what is essential is the confidence region of a set of true values which is the object of our observation at a finite number of stations. A de- 
finite set of isolines, such as shown in Fig. 3 and Fig. 7, which was drawn for the benefit of an intuitional grasp, is only an admissible candidate for the unknown true set. Owing to the finiteness of the number of stations, we cannot find the confidence region of isolines themselves, but admissible isolines may have, in general, a fairly wide sphere and it should be emphasized that the difference between two admissible sets of icolines is not significant. Accordingly, it may be inevitable and admissible that the method of drawing a representative set of isolines depends on a somewhat subjective procedure.

We could reasonably apply the method of least squares if we, in the first place, estimate the confdence region of a set of true values by our method and then we assume a functional form of spatial distribution on the results of the first step. However, it may be also unavo:dable that the functional form itself will be subjectively chosen, so long as no regulations are provided.

In our method of proportional division point, we assumed the local linearity of spatial distribution $f(\xi, \eta)$ in regard to the region for which we have no observed data. In the case where we have no information except the observed data, such assumption may be unavoidable. However, if we have a priori some information on the local distribution, we should assume a non-linear function $f(\xi, \eta)$ suitable for the local peculiarity.

\section{References}

[1]. Panofsky, H. A., 1949: Objective Weather-Map Analysis, Journal of Meteorology 6, p. 386 .

[2] Deming, W. E., 1940: Statistical Adjustment of Data, New York, Wiley.

[3] Wilks, S. S., 1943: Mathematical Statistics, New Jersey, Princeton. 